\title{
La rivista che vorrei
}

\author{
Ersilia Vita Fiscarelli \\ Ospedale Pediatrico Bambino Gesù, Roma, Italia
}

Le riviste scientifiche giocano da sempre un ruolo fondamentale nella diffusione delle conoscenze, in ogni campo disciplinare, e l'identità di una Associazione scientifica è veicolata e rafforzata anche da una propria rivista. Microbiologia Medica (MM), organo ufficiale dell'Associazione Microbiologi Clinici Italiani (AMCLI), per il fatto di essere espressione di una grande associazione professionale, costituisce una realtà particolarmente significativa nel panorama editoriale, molto variegato, della microbiologia italiana. E' una rivista a periodicità trimestrale, disponibile in formato elettronico sul sito www.microbiologiamedica.com, consultabile dai Soci nell'Area Riservata, che si propone essenzialmente finalità formative e informative. Le attività della rivista, in particolare, si concretano nella pubblicazione di articoli scientifici (revisioni, articoli originali, manoscritti, casi clinici) nel campo della microbiologia clinica, con l'obiettivo di promuovere il costante aggiornamento degli associati e favorire la diffusione e la divulgazione delle nuove conoscenze nella comunità scientifica, contribuendo ad un sapere di base che supporta la ricerca applicata e il trasferimento tecnologico. È aspirazione della rivista il massimo coinvolgimento di tutti i soci e sollecitare gli interessi sia dei lettori che degli Autori. Con la finalità di favorire la partecipazione e l'interesse negli argomenti proposti, nel mese di marzo 2018 presso gli iscritti AMCLI è stata promossa l'indagine conoscitiva "La rivista che vorrei", che, in particolare, si proponeva di investigare l'opinione dei soci riguardo a precise tematiche poste da un questionario.

Un questionario strutturato in 9 domande a risposte multiple (Materiale Supplementare) è stato inviato on-line a 1296 soci

\author{
Autore corrispondente: Ersilia Vita Fiscarelli, Ospedale Pediatrico \\ Bambino Gesù, Piazza di Sant'Onofrio 4, 00165 Roma (RM), Italia. \\ E-mail; evita.fiscarelli@opbg.net \\ Parole chiave: Microbiologia Medica, AMCLI, questionario. \\ Conflitto d'interesse: L'autore non dichiara alcun conflitto d'interesse. \\ Fondi: nessuno \\ Ricevuto per la pubblicazione: 12 December 2018. \\ Accettato per la pubblicazione: 12 December 2018. \\ (C) Copyright E.V. Fiscarelli, 2018 \\ Licensee PAGEPress, Italy \\ Microbiologia Medica 2018; 33:7997 \\ doi:10.4081/mm.2018.7997 \\ This article is distributed under the terms of the Creative Commons \\ Attribution Noncommercial License (by-nc 4.0) which permits any non- \\ commercial use, distribution, and reproduction in any medium, provid-
} ed the original author(s) and source are credited.
AMCLI. Ogni quesito prevedeva al massimo 2 risposte. Hanno aderito alla indagine compilando correttamente il questionario il $29.7 \%$ dei Soci.

Una breve sintesi delle opinioni degli iscritti. È quasi unanime (98.6\%) l'opinione che gli articoli pubblicati su riviste scientifiche on-line e corsi pratici residenziali sono gli strumenti più utili per l'aggiornamento professionale in tempo reale, sebbene circa $1 / 3$ dei partecipanti all'indagine (36\%) riconoscono che anche convegni e congressi svolgono un ruolo importante. Solo il 4\% dei microbiologi leggono tutti gli articoli pubblicati su MM, oppure si limitano a sfogliare la rivista; una percentuale di iscritti superiore all' $85 \%$ legge solo gli articoli di interesse. In generale gli argomenti trattati apportano nuove conoscenze che possono essere implementate nella pratica clinica (52\%), sebbene non tutti (39\%) ne hanno modo e/o strumenti. Esattamente la metà $(50 \%)$ dei microbiologi che hanno risposto al questionario avevano in precedenza pubblicato su MM, soprattutto articoli originali (61\%) e casi clinici (44\%), mentre l'assenza di indicizzazione della rivista è la motivazione principale per cui Il $59 \%$ dei microbiologi ha preferito pubblicare altrove. Il $36 \%$ dei partecipanti ( $1 / 3$ circa) non ha mai scritto un articolo, ma avrebbe piacere a pubblicare, e il $12 \%$ molto pessimisticamente afferma che avere pubblicazioni nel proprio CV non migliora il proprio ruolo professionale. I contributi possono essere in inglese o in italiano, indifferentemente $(50 \%)$, mentre la maggioranza dei microbiologi che hanno risposto al questionario (81\%) ritengono che MM debba pubblicare soprattutto Procedure, Protocolli, Raccomandazioni e Linee-guida, in minor misura Articoli originali (40\%), Monografie (35\%) e Case report (33\%). E infine tutti concordano per una rubrica "Risponde l'esperto".

Sono circa 50 i servizi di microbiologia clinica operanti in Italia (P. Clerici, Presidente AMCLI). In relazione soprattutto al grado di impegno assistenziale, le strutture sono caratterizzate da differenti livelli di complessità tecnica-organizzativa e di qualificazione professionale degli addetti ai "lavori". I risultati di questa indagine, che mirava a indagare $\mathrm{i}$ bisogni editoriali degli iscritti AMCLI, provvedono a fotografare un "mondo microbiologico" con bisogni editoriali molto diversificati. Emerge, in particolare, l'esigenza che la rivista pubblichi Linee-Guida, Procedure, Protocolli e Raccomandazioni, nella visione di un giornale che promuovendo le conoscenze professionali e le competenze e le abilità clinico-tecniche possa essere un "strumento utile" per un costante aggiornamento. In generale, si percepisce l'espressione di fabbisogni formativi e la consapevolezza da parte dei professionisti che operano nei Servizi di microbiologia clinica che la core competence è un requisito fondamentale per una diagnostica microbiologica di qualità e, quindi per la sicurezza dei pazienti. Un altro dato che emerge dalla indagine è che circa $1 / 3$ dei partecipanti (36\%) non ha mai pubblicato su riviste scientifiche, principalmente perchè non sempre $(12 \%)$ le pubblicazioni accademiche trovano un riconoscimento nel profilo professionale degli ope- 
ratori e anche perché ritengono di non essere preparati per la stesura di un manoscritto scientifico. Quest'ultima considerazione merita una particolare riflessione. Tra tutte le forme di comunicazione, non potendo avvalerci del tono della voce e di gesti per enfatizzare o minimizzare il contenuto, quella scritta è la più difficile da esercitare. Naturalmente non tutti abbiamo la stessa attitudine per la scrittura. Alcuni hanno un talento naturale per la collocazione delle parole all'interno delle frasi, nella costruzione efficace di un periodo; forse, la capacità di narrare scrivendo fa parte del patrimonio genetico di ciascuno di noi. Ma l'arte di scrivere, così come ogni altra arte, può essere insegnata; il metodo che ne sta alla base può essere appreso e lo stile di scrittura di una persona può essere migliorato. Le Associazioni di professionisti sanitari come
l'AMCLI, che hanno la mission di supportare tutte le attività scientifiche degli iscritti, dovrebbero accogliere anche questa istanza e riconsiderare strategie formative che siano specificamente orientate in questa direzione. I nuovi canali di comunicazione che abbiamo oggi a disposizione, opportunamente impiegati, possono essere strumenti straordinari per coltivare e intensificare le relazioni e il dialogo. Come Editor in Chief della rivista, questo sarà il mio impegno: sviluppare e consolidare questa visione. E allora, invito i Colleghi microbiologi, soprattutto i giovani che avrebbero piacere ad avvicinarsi alla scrittura scientifica, ma temono insuccessi, a utilizzare Microbiologia Medica come una "palestra", dove allenarsi all'uso delle parole. Come? scrivendo, sottomettendo, rivedendo, pubblicando. 